\title{
Object Discretization in Higher Dimensions
}

\author{
Valentin E. Brimkov ${ }^{1}$, Eric Andres ${ }^{2}$, and Reneta P. Barneva ${ }^{1}$ \\ 1 Eastern Mediterranean University, \\ Famagusta, P.O. Box 95, TRNC, Via Mersin 10, Turkey \\ \{valentin. brimkov, reneta. barneva\} @emu.edu.tr \\ 2 SIC-IRCOM, Université de Poitiers, Bat. SP2MI - BP. 179, \\ F-86960 Futuroscope Cédex - France \\ andres@sic.sp2mi.univ-poitiers.fr
}

\begin{abstract}
In this paper we study discretizations of objects in higher dimensions. We introduce a large class of object discretizations, called $k$ discretizations. This class is natural and quite general, including as special cases some known discretizations, like the standard covers and the naive discretizations. Various results are obtained in the proposed general setting.
\end{abstract}

Keywords: Object discretization, Supercover, Standard cover, Naive discretization, $k$-Discretization

\section{Introduction}

With the present paper we undertake an investigation aimed at providing a general framework for developing a discrete geometry of surfaces and bodies of arbitrary dimension. We define and study certain discretizations that generalize some well-known discretization schemes, applicable mainly to linear objects (e.g., hyperplanes). Among them, one can list some recent studies on supercovers and standard covers of hyperplanes and affine subspaces [3,1,2], as well as earlier discretization schemes (see, e.g., [18,20]).

One may expect that the theoretical and practical worth of multidimensional discrete geometry will be increasing. An argument in support of such an expectation is provided by some recent applications. In fact, 4-dimensional imaging is already existing, due to, e.g., PET scans and other dynamic medical images. Further applications are to be expected in multimedia where hypertexts together with images, motion video and sound are manipulated. Another motivation is inspired by mathematical arguments. For every Euclidean notion we may have numerous corresponding discrete notions, depending on the applications and the Euclidean property we want to preserve. The great diversity of possible discretizations is due to their discrete nature. If we consider objects and properties (especially topological properties) only in $2 \mathrm{D}$ or $3 \mathrm{D}$, we will not necessarily choose definitions that would have natural extensions in higher dimensions later on, in case we need to go to higher dimensions (for animation, for instance). In other words, a geometry that is not coherent in all dimensions cannot serve as a 
good ground for future developments. More general results in higher dimensions will give insight to the practically important $2 \mathrm{D}$ and $3 \mathrm{D}$ versions of the theory, and may facilitate the deeper understanding of the matter. Thus, developing a reasonable $n$-dimensional discrete geometry is not simply an academic exercise, but is dictated by real needs.

We rely implicitly on the existing knowledge in the digital topology (see, e.g., $[15,16,14,8])$. In particular, we conform to certain widely adopted terminology, which we briefly recall in Section 2 . In Section 3 we define a minimal cover of a body and study some basic properties of such covers. In Section 4 we introduce the fundamental concept of $k$-discretization and $k$-cover of surfaces. We also expose a relation between the $k$-discretizations and some other discretizations based on Hausdorff distance [21,19]. In Section 4.3 we present some results about connectivity of $k$-discretizations, while in Section 4.4 we illustrate some of the new notions and results in the case of hyperplanes and halfspaces. In particular, the 0-discretizations of hyperplanes appear to be standard discrete planes. The latter have been studied in the framework of the arithmetic geometry $[2,17]$.

\section{Preliminaries}

We recall some basic notions to be used in the sequel. To obtain more details about some of them, the reader is referred to [9].

A discrete (resp. Euclidean) point $p$ is an element of $Z^{n}$ (resp. $R^{n}$ ). Throughout we will assume that $n \geq 2$. By $p_{i}$ we denote the $i$ th coordinate of $p$. A discrete (resp. Euclidean) object is a set of discrete (resp. Euclidean) points.

Let $p \in Z^{n}$. A voxel with center $p$ is the unit cube $V(p)=\left[p_{1}-\frac{1}{2}, p_{1}+\right.$ $\left.\frac{1}{2}\right] \times \ldots \times\left[p_{n}-\frac{1}{2}, p_{n}+\frac{1}{2}\right]$. A $j$-dimensional facet of $V(p)$ will be referred as to a $j$-facet, $0 \leq j \leq n^{1}$. We also define $V(A)=\cup_{p \in A} V(p)$, i.e., $V(A)=\left\{x \in R^{n}\right.$ : $x \in V(p)$ for some $p \in A\}$.

The Euclidean points $p=\left(p_{1}, \ldots, p_{n}\right)$ and $q=\left(q_{1}, \ldots, q_{n}\right)$ are said to be $k$ neighbors, with $0 \leq k \leq n$, if $\left|p_{i}-q_{i}\right| \leq 1$ for $1 \leq i \leq n$, and $k \leq n-\sum_{i=1}^{n}\left|p_{i}-q_{i}\right|$. A $k$-path in a discrete object $A$ is a sequence of discrete points from $A$ such that every two consecutive points are $k$-neighbors. $A$ is called $k$-connected if there is a $k$-path connecting any two points of $A$. A $k$-component is a maximal $k$ connected subset of $A$.

Let $D$ be a subset of a discrete object $A$. If $A-D$ is not $k$-connected then the set $D$ is said to be $k$-separating in $A$.

Now we introduce some other notions. Let $M \subseteq R^{n}$ be a Euclidean object. For technical convenience throughout the paper we will assume that $M$ is a closed set. A point $p \in R^{n}$ is called boundary point for $M$ if it is neither internal nor external for $M$. The set of all boundary points for $M$ is called boundary of $M$ and denoted $B d(M)$. Since $M$ is closed, $B d(M) \subseteq M$.

A cover of $M$ is any set of voxels $C(M)$ whose union contains $M$. The supercover of $M$ is the set $S(M)$ of all voxels which are intersected by $M$. Clearly,

\footnotetext{
1 Thus the 0 -facets are the voxel vertices, the 1 -facets - its edges, the $(n-1)$-facets are the voxel faces, and the $n$-facet is the voxel itself.
} 


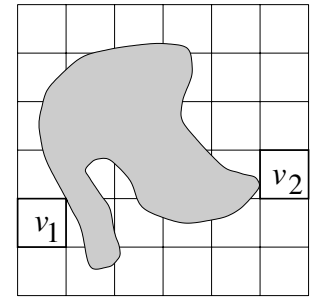

a)

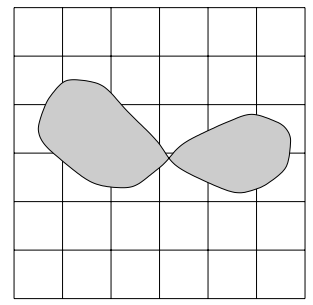

b)

Fig. 1. a) Example of an object that is a body in $R^{2}$. The voxels $v_{1}$ and $v_{2}$ are osculant for the object. b) Example of an object that is not a body. It has no osculant voxels

each of the following inclusions is possible for a cover $C(M)$ and a supercover $S(M)$ : (i) $S(M) \subseteq C(M)$; (ii) $C(M) \subseteq S(M)$. In our study we will be interested in covers of Type (ii), which are "tighter" than or identical to supercovers. We will consider covers and other discretizations that are not necessarily covers, of Euclidean sets with topological dimension $h$, where $0 \leq h \leq n$. In particular, we will consider covers of bodies. We will say that a closed set $B \subseteq R^{n}$ is a body if it is simply connected ${ }^{2}$, has a topological dimension $\operatorname{dim}(B)=n$, and for every point $p \in B d(B), B-\{p\}$ is a simply connected set. For example, the set in Figure 1a is a body in $R^{2}$, while the one on Figure $1 \mathrm{~b}$ is not. We will also study discretizations of surfaces of different dimension, in particular, $(n-1)$-dimensional surfaces. We will suppose that the surface is given in explicit form $\Gamma=\left\{x \in R^{n}: x_{n}=f\left(x_{1}, \ldots, x_{n-1}\right)\right\}$, where $f$ is a continuous function defined on $R^{n-1}$.

We will call a voxel $V(p)$ osculant for a Euclidean object $M$ if $V(p) \cap M$ is contained in a $j$-facet of $V(p)$, for $0 \leq j<n$ (Figure 1a). An $n$-bubble in $R^{n}$ is a set of $2^{n}$ voxels sharing a common vertex $Q$. Equivalently, an $n$-bubble can be considered as the supercover of a half-integer point, namely the point $Q$ in the alternative definition.

We conclude this section with the following lemma, which will be used in the next section.

Lemma 1. Let $M \subseteq \mathbf{R}^{n}$ be an arbitrary connected Euclidean set. Then $S(M)$ is $(n-1)$-connected.

A proof can be obtained as a straightforward generalization of the proof for the case $n=3$ [9]. For a detailed accounting on various other properties of supercovers we refer to [1]. In particular, it is well-known that a supercover is not, in general,

\footnotetext{
${ }^{2}$ We recall that a set $M \subseteq R^{n}$ is called simply connected if it is homeomorphic to the unit ball in $R^{n}$.
} 


\section{Minimal Covers}

In this section we consider a natural and important class of covers.

Definition 1. Let $M$ be a Euclidean object. We call its cover $C(M)$ minimal if there is no other cover of $M$ that is a proper subset of $C(M)$.

Next we study some properties of the minimal covers.

Proposition 1. If a cover of an object $M$ does not contain any osculant voxel, then it is minimal.

Proof Let $C(M)$ be a cover of $M$ that does not contain osculant voxels, and let $v$ be an arbitrary voxel from $C(M)$. By definition of osculant voxel, the intersection $M_{v}=v \cap M$ is not contained in a facet of $v$, and therefore is also not contained in a facet of any other voxel. Let us remove $v$ from $C(M)$. The remaining set of voxels $C(M)-\{v\}$ does not contain $M_{v}$, since a set cannot be contained in two distinct voxels at the same time, unless it is contained in a common facet of theirs. Thus $C(M)-\{v\}$ is not a cover of $M$. Hence the cover $C(M)$ is minimal.

In the case of bodies, the condition of the above theorem completely characterizes the minimal covers, as the following theorem suggests.

Proposition 2. A cover of a body (not necessarily bounded) is minimal if and only if it does not contain any osculant voxels.

Proof One direction of the proof follows from Proposition 1. We prove the other direction. Let $B$ be a body and $C(B)$ its minimal cover. Assume that $C(B)$ contains a voxel $v$ which is osculant for $B$. Then we have $B_{v}=v \cap B \subseteq F$, where $F$ is a facet of $v$.

If $C(B)$ contains another voxel $v^{\prime} \supseteq B_{v}$, then $C(B)-\{v\}$ is still a cover of $B$ and thus $C(B)$ is not minimal. Let us assume that $B_{v}$ is not contained in any voxel of $C(B)$ other than $v$. Note that $B_{v}$ cannot be a single point. The opposite would be possible either if $B_{v}$ belongs also to another voxel (one containing the rest of $B_{v}$ or a part of it) or if $B=B_{v}$, which is impossible since $B$ is a body.

We distinguish between two cases. Let us first assume that $B_{v}=B$. Since $B_{v}$ is contained in a facet of $v$ with topological dimension less than or equal to $n-1$, the same follows for the dimension of $B$, which contradicts the fact that $B$ is a body. The other possibility is $B=B_{v} \cup B^{\prime}$ for some set $B^{\prime} \neq \emptyset$, such that $B_{v} \cap B^{\prime}=\emptyset$. Since $B_{v}$ and $B^{\prime}$ are disjoint and $B$ is simply connected, $B_{v}$ is simply connected, as well. Since $0 \leq \operatorname{dim}\left(B_{v}\right) \leq n-1$, every point of $B_{v}$ belongs to $B d(B)$. Then there will be a point $p \in B_{v}$ such that the set $B_{v}-\{p\}$ is not simply connected, which contradicts the definition of body.

Proposition 3. A body $B$ has a unique minimal cover.

Proof Consider the supercover $S(B)$ of $B$, and let us remove from it all voxels which are osculant for $B$. Having in mind the proof of Proposition 2, we can conclude that the obtained set of voxels is a minimal cover, which is clearly unique. 


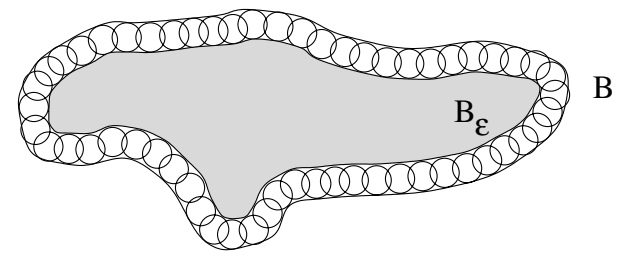

Fig. 2. Illustration to the proof of Theorem 1

Theorem 1. The minimal cover of a body $B$ is $(n-1)$-connected. It can be obtained from the supercover of $B$ by removing all osculant voxels for $B$.

Proof Let $S(B)$ be the supercover of a body $B$, and let us remove from it all the voxels that are osculant for $B$. Denote the resulting set of voxels by $C(B)$. By Propositions 2 and $3, C(B)$ is the unique minimal cover of $B$. We will show that $V(B)$ is $(n-1)$-connected. For this, let us consider the set $C l\left(B_{\varepsilon}\right) \subset B$, which is the closure of the set $B_{\varepsilon}$ obtained from $B$ by excluding the union of all the closed balls with diameter $\varepsilon$ which are contained in $B$ and have a single common point with the boundary of $B$ (see Figure 2). Since $B$ is a body, for $\varepsilon$ small enough (e.g., $\varepsilon<1 / m$ for enough large integer $m$ ) $B_{\varepsilon}$ will be a body as well, and thus a connected subset of $R^{n}$. It is also clear that for the so defined $\varepsilon$, the set $C(B)$ will be a supercover of $B_{\varepsilon}$. Then, by Lemma $1, C(B)$ is $(n-1)$-connecded as a supercover of a connected set.

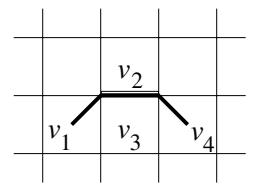

a)

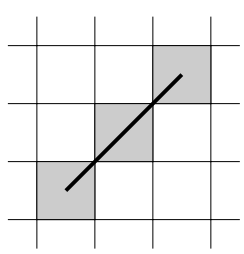

b)

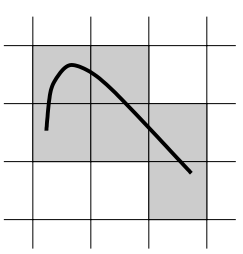

c)

Fig. 3. a) The curve has two minimal covers: $\left\{v_{1}, v_{2}, v_{4}\right\}$ and $\left\{v_{1}, v_{3}, v_{4}\right\}$. Each of them contains a voxel that is osculant for the curve ( $v_{2}$ and $v_{3}$, respectively). b) The segment on the figure has a minimal cover that is not $(n-1)$-connected.

c) The minimal cover of the curve contains a 2-bubble

With surfaces, Propositions 2, 3, and Theorem 1 do not hold, in general, as illustrated in Figures $3 \mathrm{a}$ and b. A minimal surface cover may contain osculant voxels (Figure 3a), as well as bubbles (Figure 3c). 


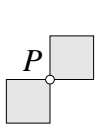

a1)

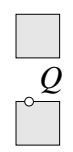

a2)

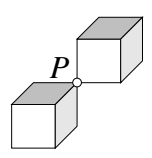

b1)

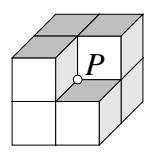

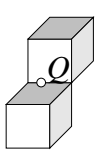

b2)
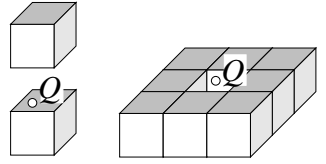

b3)

Fig. 4. Illustration to Definition 2. In dimension 2: a1) 0-tunnel; a2) 1-tunnel. In dimension 3: b1) 0-tunnel; b2) 1-tunnel; b3) 2-tunnel

\section{Surface Discretizations}

In this section we are concerned with discretizations of surfaces of different dimensions. We introduce and study a large class of discretizations called $k$ discretizations. For this purpose, we first introduce a definition of tunnels in discrete objects.

\subsection{Tunnels in Discrete Objects}

Bellow we suggest a definition of tunnels, applicable to a broad class of discrete objects. Let $A \subseteq R^{n}$ be a discrete object, such that the set $R^{n}-V(A)$ is connected. (This condition ensures that there are no "caves" in $V(A)$.)

Definition 2. If the set $V(A)$ is not simply connected, we postulate that $A$ has a $j$-tunnel, for every $0 \leq j \leq n-1$.

0-tunnels: If $n=2$, then $A$ has a 0-tunnel if $A$ contains two voxels whose intersection is their common vertex and no other voxel from $A$ shares the same vertex (Figure 4 a1). If $n>2$, then $A$ has a 0 -tunnel either if $A$ contains two voxels whose intersection is their common vertex and no other voxel from $A$ shares the same vertex, or if there is a voxel $v \in A$ and a vertex $P$ of $v$ with $P \in B d(V(A))$, such that $V(A)-\{P\}$ is not a simply connected set (Figure 4 b1).

$j$-tunnels, $1 \leq j \leq n-1: A$ has a $j$-tunnel if there is a voxel $v \in A$ and a $j$-facet $F$ of $v$, such that $F \subseteq B d(V(A))$, and $V(A)-\{Q\}$ is not a simply connected set, for every point $Q$ belonging to the relative interior ${ }^{3}$ of $F$. Thus if $A$ is disconnected, it has $(n-1)$-tunnels. (See Figure 4 a2,b2,b3.)

A discrete object without $k$-tunnels is called $k$-tunnel-free.

\section{$4.2 k$-Discretizations and $k$-Covers}

We start with recalling the definition of Hausdorff distance. Let $E$ be a metric space with metric $d$, and $\mathcal{E}$ a family of closed non-empty subsets of $E$. For every $x \in E$ and every $A \in \mathcal{E}$ let $d(x, A)=\inf \{d(x, y): y \in A\}$. Then, given two sets

\footnotetext{
${ }^{3}$ Relative interior of a $j$-facet $F$ is the interior of $F$, considered as a subset of $R^{j}$.
} 


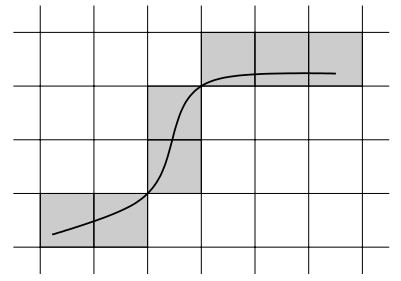

a)

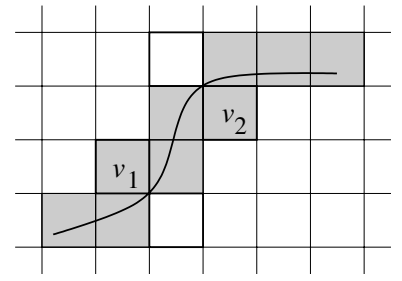

b)

Fig. 5. Illustration to Definition 3. a) 1-Discretization. b) 0-Discretization. It can be obtained from the 1-discretization by adding the voxels $v_{1}$ and $v_{2}$

$A, B \in \mathcal{E}, H_{d}(A, B)=\max \{\sup \{d(a, B): a \in A\}, \sup \{d(A, b): b \in B\}\}$ is called the Hausdorff distance between $A$ and $B$.

Let a surface $\Gamma \subset R^{n}$ be given, with topological dimension $h, 0 \leq h \leq n-1,{ }^{4}$ i.e., $\Gamma=\left\{x \in R^{n}: x_{i}=f_{i}\left(t_{1}, \ldots, t_{h}\right), 1 \leq i \leq n, t_{j} \in R, 1 \leq j \leq h\right\}$ where $f_{i}$ are continuous functions of the variables $t_{1}, \ldots, t_{h}$. In a very general sense, we will consider every subset of the supercover $S(\Gamma)$ as a discretization of $\Gamma$. Of interest for us are discretizations that are $(n-1)$-tunnel-free (i.e., without "holes"). Let $D(\Gamma) \subseteq S(\Gamma)$ be a $k$-tunnel-free discretization of $\Gamma$. We will call a discrete point $p \in D(\Gamma) k$-simple, if $D(\Gamma)-\{p\}$ is still $k$-tunnel-free. If a $k$-tunnel-free discretization $D(\Gamma)$ does not contain $k$-simple points, we will call it $k$-minimal ${ }^{5}$. Now we are able to give the following definition.

Definition 3. Let $S(\Gamma)$ be the supercover of $\Gamma$, and $\mathcal{D}_{k}(\Gamma)$ the family of all subsets of $S(\Gamma)$ that are $k$-minimal, for some $0 \leq k \leq n-1$. We will call a set of voxels $D_{k}(\Gamma) \in \mathcal{D}_{k}(\Gamma)$ a $k$-discretization of $\Gamma$ if it is $(n-1)$-tunnel-free and the Hausdorff distance $H_{d}\left(\Gamma, V\left(D_{k}(\Gamma)\right)\right)$ is minimal, over all the elements of $\mathcal{D}_{k}(\Gamma)$.

Further we consider mainly the case when $d$ is the Euclidean metric in $R^{n}$. Some illustrations to Definition 3 are presented in Figure 5. We remark that in this definition the supercover of a surface is used as a natural upper bound for the amount of voxels included in a $k$-discretization.

Clearly, for $1 \leq k \leq n-1$, a $k$-discretization of a surface is not necessarily a cover of its, while a 0 -discretization always is. If $\Gamma \subseteq D_{k}(\Gamma)$, we call the discretization $D_{k}(\Gamma)$ a $k$-cover of $\Gamma$. One can observe that the maximal $k$ for

\footnotetext{
${ }^{4}$ For example, if $h=0, \Gamma$ is a Euclidean point, whereas if $h=1, \Gamma$ is a curve in $R^{n}$.

${ }^{5}$ Traditionally, the following definitions are used. Let $B$ be $k$-separating in a discrete object $A$ and $A-B$ have exactly two connected components. A $k$-simple point of $B$ is a point $p \in B$ such that $B-p$ is still $k$-separating. A $k$-separating discrete object is called $k$-minimal if it does not contain any $k$-simple point. The advantage of our version of the definition of $k$-minimality is that it applies also to an object that is not $k$-separating in another object.
} 
which a $k$-discretization of a surface appears to be a cover, corresponds to a minimal cover as defined in the previous section.

Of a special interest are the two extreme cases $k=0$ and $k=n-1$. As a matter of fact, 0 - and $(n-1)$-discretizations have been studied in the special cases when $\Gamma$ is a straight line or plane. Among the earliest works is the one of Bresenham who proposes an algorithm to discretize a straight line in the plane [5]. Reveillès [17] considers Bresenham lines in the framework of the arithmetic geometry. He shows that the Bresenham line, called also naive line, admits an analytical description. 3D naive planes and lines are also defined and studied $[10,12,13,11]$. The naive lines/planes are proved to be the thinnest possible discretizations that approximate best the corresponding Euclidean lines/planes. It is easy to observe that they are $(n-1)$-discretizations. Naive discretizations have also been studied in the case of hyperplanes. Stojmenovic et al. [18] propose a scheme for discretizing hyperplanes. Further related results within the Stojmenovic scheme are obtained in [20]. In [3] it is shown that the Stojmenovic discretization is an $n \mathrm{D}$ analog of naive discretization. As in the $2 \mathrm{D}$ and $3 \mathrm{D}$ cases, a naive discretization of a hyperplane appears to be an $(n-1)$-discretization.

0-Discretizations of 2D lines and of planes are also well-studied. Such sort of discretizations have been defined and investigated in analytical setting $[17,10]$, and are known as standard discretizations. In higher dimensions, standard discretizations are considered in [2].

Definition 3 provides a structural framework for studying naive $(n-1)$ discretizations and standard 0-discretizations for arbitrary surfaces. It also gives a more general view on the matter, considering the naive and the standard discretizations as (extreme) particular cases within the class of $k$-discretizations. One can expect that various $k$-discretizations with $k \neq 0, n-1$ may be interesting for certain applications.

We remark that discretizations based on the concept of Hausdorff distance have been proposed in $[21,19]$. More precisely, a Hausdorff discretization of a compact set $K \subset R^{n}$ is defined as the union of all subsets of $Z^{n}$ minimizing the Hausdorff distance to $K$. One can observe that all the naive $(n-1)$-discretizations that are in a minimal Hausdorff distance from a Euclidean object contribute to its Hausdorff discretization.

The Hausdorff and the $k$-discretization elucidate discretization issues from different angle. The former one is mathematically elegant and has some interesting properties, see [19, Sections 3,4]. Besides, interesting relations hold between the Hausdorff discretization and other well-known discretizations. For instance, under certain conditions, the Hausdorff discretization is equivalent to the supercover discretization defined above [19, Theorem 2].

On the other hand, with the study of $k$-discretizations we pursue deeper understanding of the structure of some natural discretizations (like the naive and the standard discretizations) within a more general setting. The $k$-discretizations can be considered as the thinnest possible discretizations without tunnels of certain type, while the Hausdorff discretizations, in general, can be seen to 
be thicker. In this sense, our approach is much in the spirit of some recent work $[7,6,4]$ on thin polyhedral discretizations.

\subsection{Connectivity of $k$-Discretizations}

In this section we report our basic result about connectivity of $k$-discretizations of surfaces.

Theorem 2. Let $\Gamma \subset R^{n}$ be an $h$-dimensional surface, $0 \leq h \leq n-1$. A $k$ discretization of $\Gamma$ with $0 \leq k \leq n-1$ is $\max \{h-1, n-k-1\}$-connected.

Idea of the Proof Let $D_{k}(\Gamma)$ be a $k$-discretization of $\Gamma$. Let $v^{\prime}$ and $v^{\prime \prime}$ be arbitrary two voxels from $D_{k}(\Gamma)$. Since $D_{k}(\Gamma)$ is connected, there is an ordered set of voxels $\left\{v_{1}, v_{2}, \ldots, v_{l}\right\}$ with $v_{1}=v^{\prime}, v^{\prime \prime}=v_{l}$, such that any two consecutive elements $v_{i}, v_{i+1}, 1 \leq i \leq l-1$, are at least 0 -connected. To obtain a proof of the theorem, it suffices to show that $v_{i}$ and $v_{i+1}$ are $(h-1)$ - and $(n-k-1)$-connected.

1. $(h-1)$-connectivity. Let $F$ be the facet of maximal dimension $f, 0 \leq f \leq$ $n-1$, so that $F$ is common for $v_{i}$ and $v_{i+1}$. If $f \geq h-1$, then $v_{i}$ and $v_{i+1}$ are $(h-1)$-connected. Suppose that $f<h-1$. Let $W$ be the set of all voxels that share the facet $F$. One can show that there is an ordered set of voxels $W^{\prime}=\left\{v_{1}, v_{2}^{\prime}, \ldots, v_{m}^{\prime}, v_{l}\right\}, m<l, W^{\prime} \subseteq W$, so that any two consecutive voxels in $W^{\prime}$ are $(h-1)$-connected. If one assumes the opposite, one can show that there must be an $(n-1)$-tunnel in $D_{k}(\Gamma)$, which contradicts the definition of $k$ discretization. The proof is based on a detailed analysis of the structure of the multidimensional discrete space, and will be included in the full journal version of the paper.

2. $(n-k-1)$-connectivity. By definition, if $D_{k}(\Gamma)$ is $k$-minimal, then it is $k$ tunnel-free. Then one can show that if $D_{k}(\Gamma)$ is not $(n-k-1)$-connected, it will contain $k$-tunnels. The details will be included in the full paper.

The above theorem implies a number of corollaries about certain important special classes of surfaces.

Corollary 1. Let $\Gamma$ be an $(n-1)$-dimensional surface in $R^{n}$.

(a) For any $1 \leq k \leq n-1$, a $k$-discretization of $\Gamma$ is $(n-2)$-connected. Thus, in particular, any naive $(n-1)$-discretization is $(n-2)$-connected.

(b) Any standard cover (0-discretization) of $\Gamma$ is $(n-1)$-connected.

For the case $h=1$, i.e., when the surface is a curve, we obtain the following corollary.

Corollary 2. A $k$-discretization of a curve in $R^{n}$ is $(n-k-1)$-connected. (In particular, a standard 0-discretization is $(n-1)$-connected, and a naive $(n-1)$ discretization is 0-connected, see Figure 6a.)

In the case of a naive $(n-1)$-discretization we have the following corollary.

Corollary 3. A naive ( $n-1)$-discretization of a $h$-dimensional surface is $(h-1)$ connected. (Thus a naive $(n-1)$-discretization of an $(n-1)$-dimensional surface is $(n-2)$-connected, in accordance with Corollary 1, see Figure 6b.) 


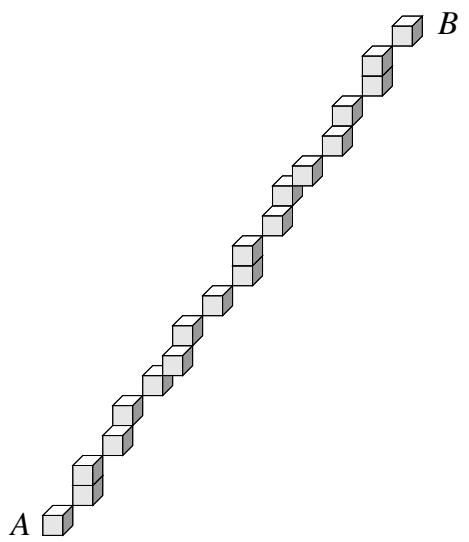

a)

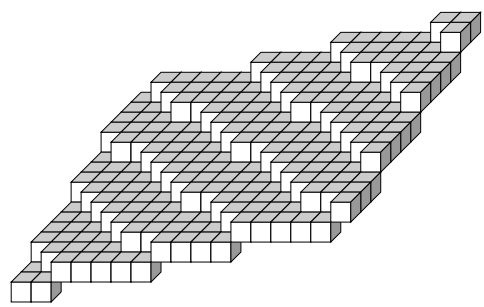

b)

Fig. 6. Illustrations to Corollaries 2 and 3. a) A portion of naive 2-discretization of the Euclidean line between the points $A=(0,0,0)$ and $B=(11,13,18)$. It has 0 -tunnels and is 0 -connected. b) A portion of naive 2-discretization of the Euclidean plane $-4 x-9 y+18 z=0$. It has 1 -tunnels and is 1 -connected

\subsection{Applications: $k$-Discretizations of Hyperplanes and Halfspaces}

In this section we illustrate the concept of $k$-discretizations in the important case when the surface is a hyperplane. We recall that an arithmetic plane $e^{6} P=$ $P\left(\beta, \alpha_{1}, \alpha_{2}, \ldots, \alpha_{n}, \omega\right)$ is defined as the set of integer points which satisfy the double linear Diophantine inequality $0 \leq \beta+\sum_{i=1}^{n} \alpha_{i} x_{i}<\omega$.

Let $H: b+\sum_{i=1}^{n} a_{i} x_{i}=0$ be a hyperplane. Let $j$ be the least index for which $a_{j} \neq 0$, and let $a_{j}>0^{7}$. Then a standard cover of $H$ has been defined as

$$
S t(H)=\left\{x \in Z^{n}:-\frac{\sum_{i=1}^{n}\left|a_{i}\right|}{2} \leq b+\sum_{i=1}^{n} a_{i} x_{i}<\frac{\sum_{i=1}^{n}\left|a_{i}\right|}{2}\right\} .
$$

It is proved [2, Proposition 13] that $S t(H)$ is indeed a cover, which is $(n-1)$ connected, 0-minimal, and $n$-bubble-free. One can show that the Hausdorff distance between $H$ and $S t(H)$ is the minimal possible over all 0-tunnel-free discretizations of $H$. This, together with the 0 -minimality, implies that the above definition is in accordance with the global definition of a 0-discretization (standard cover) given in the previous section. More precisely, we can state the following theorem.

Theorem 3. The set of voxels determined by Condition (1) is a 0-discretization (standard cover) of the hyperplane $H: b+\sum_{i=1}^{n} a_{i} x_{i}=0$.

\footnotetext{
${ }^{6}$ Sometimes also called discrete analytical plane.

${ }^{7}$ Then the hyperplane $H$ is said to have a standard orientation [2].
} 
This result can be extended to arbitrary $k$-discretizations. As a first step we reveal which arithmetic planes are $k$-discretizations. In [3] the connection between the tunnels of arithmetic hyperplanes and their thickness is investigated, and the following basic result is obtained ${ }^{8}$.

Theorem 4. [3, Proposition 9] Let $P=P\left(b, a_{1}, a_{2}, \ldots, a_{n}, \omega\right)=\left\{x \in Z^{n} \mid 0 \leq\right.$ $\left.b+\sum_{i=1}^{n} a_{i} x_{i}<\omega\right\}$ be a discrete hyperplane, where $b \geq 0, a_{i} \geq 0$ for all $i$, and $a_{i} \leq a_{i+1}$ for $1 \leq i \leq n-1$. Then, if $\omega<a_{n}$, the hyperplane has $(n-1)$ tunnels; For $0<k<\bar{n}$, if $\sum_{i=k+1}^{n} a_{i} \leq \omega<\sum_{i=k}^{n} a_{i}$, the hyperplane has $(k-1)$-tunnels and is $k$-separating; If $\omega \geq \sum_{i=1}^{n} a_{i}$, the hyperplane is tunnelfree.

The next theorem characterizes the arithmetic planes that are $k$-discretizations.

Theorem 5. Let $P$ be a discrete hyperplane as in Theorem 4. If $\omega=\sum_{i=k+1}^{n} a_{i}$, then $P$ is a $k$-discretization.

The proof will be included in the full paper. The notion of surface $k$-discretization or $k$-cover can be extended to subsets of $R^{n}$ with topological dimension $n$. For this, one can use the corresponding discretization of the boundary of the given set. Let us consider, for instance, a half-space $E$ determined by a linear inequality $a_{1} x_{1}+a_{2} x_{2}+\ldots+a_{n} x_{n} \leq b$. One can obtain a $k$-discretization of $E$ as $D_{k}(E)=\left\{x \in Z^{n}: b+\sum_{i=1}^{n} a_{i} x_{i}<\omega\right\}$, where $\omega=\sum_{i=k+1}^{n} a_{i}$, for a standard discretization convention (see [2] for getting acquainted with the notion of discretization convention). In particular, the set of voxels $S t(E)=\left\{x \in Z^{n}\right.$ : $\left.b+\sum_{i=1}^{n} a_{i} x_{i}<\omega\right\}$, with $\omega=\sum_{i=1}^{n} a_{i}$, is a 0 -discretization (standard cover) of $E$.

\section{Concluding Remarks}

In this paper we have studied minimal covers of bodies and $k$-discretizations of surfaces of arbitrary dimension. A further direction of research can be seen in studying minimal covers and $k$-discretizations of some important special classes of Euclidean objects, like affine subspaces of arbitrary dimension $0 \leq m \leq n-1$ ( $m$-flats), simplexes or some other interesting classes of polytopes, spheres, etc.

It would also be interesting to study the properties of $k$-discretizations for different choices of a metric $d$ in the definition of Hausdorff distance.

\section{Acknowledgments}

We thank the Referees for several valuable comments and suggestions.

\footnotetext{
${ }^{8}$ In the special case of discrete hyperplanes, a $j$-tunnel can be defined as follows. A discrete plane $P=P\left(\beta, \alpha_{1}, \alpha_{2}, \ldots, \alpha_{n}, \omega\right)$ has a $j$-tunnel if there are two $j$-neighboring voxels $v=\left(v_{1}, \ldots, v_{n}\right)$ and $w=\left(w_{1}, \ldots, w_{n}\right)$ such that $\alpha_{1} v_{1}+\ldots+\alpha_{n} v_{n}+\beta<0$ and $\alpha_{1} w_{1}+\ldots+\alpha_{n} w_{n}+\beta \geq 0$ [17]. It is not difficult to see that this definition is in accordance with the more general Definition 2.
} 


\section{References}

1. Andres, E.: An m-Flat Supercover is a Discrete Analytical Object. TR 1998-07, IRCOM-SIC, Université de Poitiers, Bat. SP2MI - BP. 179, F-86960 Futuroscope Cédex - FRANCE (1998) 210, 212

2. Andres, E.: Standard Cover: a New Class of Discrete Primitives. TR 1998-02, IRCOM-SIC, Université de Poitiers, Bat. SP2MI - BP. 179, F-86960 Futuroscope Cédex - FRANCE (1998) 210, 211, 217, 219, 220

3. Andres, E., Acharya, R., Sibata, C.: Discrete Analytical Hyperplanes. Graphical Models and Image Processing, 59 (5) (1997) 302-309 210, 217, 220

4. Barneva, R. P., Brimkov, V. E., Nehlig, P.: Thin Discrete Triangular Meshes. Theoretical Computer Science 264 (1-2) (2000) 73-105 218

5. Bresenham, J. E.: Algorithms for Computer Control of a Digital Plotter. IBM Syst. J., 4 (1) (1965) 25-30 217

6. Brimkov, V. E., Barneva, R. P.: Graceful Planes and Lines, Theoretical Computer Science, Elsevier, to appear 218

7. Brimkov, V. E., Barneva, R. P., Nehlig, P.: Minimally Thin Discrete Triangulations. In: Volume Graphics, M. Chen, A. Kaufman, R. Yagel (Eds.), Springer Verlag (2000) 51-70 218

8. Bertrand, G., Couprie, M.: A Model for Digital Topology. Lecture Notes in Computer Science, Vol. 1568, Springer-Verlag, Berlin (1999) 229-241 211

9. Cohen, D., Kaufman, A.: Fundamentals in Surface Voxelization. CGVIP Graphical Models and Image Processing, 57 (6) (1995) 211, 212

10. Debled-Rennesson, I., Reveillès, J-P.: A new approach to digital planes. In: SPIE Vision Geometry III, Boston 23561994217

11. Figueiredo, O., Reveillès, J-P.: A Contribution to 3D Digital Lines. In: Proc. the 5th Internat. Workshop DGCI, Clermont-Ferrand (1995) 187-198 217

12. Françon, J.: Discrete Combinatorial Surfaces. CGVIP Graphical Models and Image Processing, 57 (1995) 217

13. Kaufman, A., Shimony, E.: 3D Schan-Conversion Algorithms for Voxel-Based Graphics. In: Proc. 1986 Workshop on Interactive 3D Graphics, Chapel Hill, NC, ACM, New York (1986) 45-75 217

14. Kong, T. Y., Kopperman, R., Meyer, P. R.: A Topological Approach to Digital Topology. American Mathematical Monthly, 38 (1991) 901-917 211

15. Kong, T. Y., Rosenfeld, A.: Digital Topology: Introduction and Survey. Computer Vision, Graphics, and Image Processing 48 (1989) 357-393 211

16. Kovalevsky, V. A.: Finite Topology as Applied to Image Analysis. Computer Vision, Graphics, and Image Processing 46 (1989) 141-161 211

17. Reveillès, J.-P.: Géométrie Discréte, Calcul en Nombres Entiers et Algorithmique. Thèse d'État, Université Louis Pasteur, Strasbourg, France (1991) 211, 217, 220

18. Stojmenovic, I., Tosic, R.: Digitization Schemes and the Recognition of Digital Straight Lines, Hyperplanes and Flats in Arbitrary Dimensions. Digital Geometry. Contemporary Math. Series, Amer. Math. Soc. Providence, RI 119 (1991) 197-212 210, 217

19. Tajine, M., Wagner, D., Ronse, C.: Hausdorff Discretizations and its Comparison to Other Discretization Schemes. Lecture Notes in Computer Science, Vol. 1568, Springer-Verlag, Berlin (1999) 399-410 211, 217

20. Veerlaert, P.: On the Flatness of Digital Hyperplanes. J. Math Imaging Vision 3 (1993) 205-221 210, 217

21. Wagner, D., Tajine, M., Ronse, C.: An Approach to Discretization Based on Hausdorff Metric. ISMM'98, Kluwer Academic Publisher (1998) 67-74 211, 217 